\title{
Hysterectomy and bilateral salpingoophorectomy in female-to-male transsexual: does testosterone induce anatomopatological changes? A retrospective study
}

\author{
Marina Martin Pereda \\ Hospital Regional Universitario de Málaga \\ Maria Amores Vergara \\ Hospital Regional Universitario de Málaga \\ Lorena Sabonet Morente \\ Hospital Regional Universitario de Málaga \\ Ernesto Gonzalez Mesa \\ Hospital Regional Universitario de Málaga \\ Pilar Espejo Reina \\ Hospital Regional Universitario de Málaga \\ Jesus S. Jimenez Lopez ( $\nabla$ jesuss.jimenez.sspa@juntadeandalucia.es ) \\ Hospital Regional Universitario de Málaga
}

\section{Research Article}

Keywords: testosterone, atrophic endometrium, female-to-male transsexual, hysterectomy, malignancy

Posted Date: May 26th, 2021

DOI: https://doi.org/10.21203/rs.3.rs-539814/v1

License: (c) (1) This work is licensed under a Creative Commons Attribution 4.0 International License.

Read Full License 


\section{Abstract \\ Background}

Testosterone replacement treatment as part of gender reassignment therapy has repercussions on the final pathological result of the hysterectomies that are performed. The objective of this review is to analyze the surgical pieces of hysterectomies performed on FTM patients (female-to-male) and to describe possible changes related to testosterone. There are few studies and with a low number of patients where this possible repercussion is addressed.

\section{Methods}

A retrospective study was carried out, which included 117 patients between the ages of 21 and 56, operated on between 2010 and 2019, with at least an average duration of treatment of 5 years before gender reassignment surgery. Main outcome measure were changes in follicle stimulating hormone (FSH), luteinizin hormone (LH), testosterone (TST) and estradiol in blood, both preoperatively and postoperatively. Also the differente types of endometrium and ovaries found in histerectomy pieces.

\section{Results}

the pathological study revealed that $95(80.3 \%)$ cases showed an atrophic endometrium, 7 (6\%) cases an endometrium with secretory-type changes, and 15 (13.7\%) cases with weakly proliferating endometrium.

\section{Conclusions}

Our data revealed that the most frequent histology after chronic administration of parenteral testosterone was such compatible with atrophic endometrium, coinciding with what has been published in the literature up to now. More long-term studies on the effects of parenteral testosterone are still needed.

\section{Background}

The assignment of an individual to a specific gender, male or female, is largely based on the phenotype of the genitalia at birth, which is determined by the $X$ and $Y$ chromosomes. However, there are currently many other terms and concepts that have little to do with do with this simple classification. Terms such as sex, gender, sexual expression, sexual orientation, or sexual identity should not be confused. The latter term refers to how a person lives or experiences their gender, which may or may not coincide with their phenotypic sex. When a person identifies with their same phenotypic gender, we speak of cisgender, and when not, of transgender. Transgender people are classified into 3 main categories: trans men, trans women and non-binary (genderqueer, agender, bigender and gender-fluid) [1]. 
The present article focuses on trans men, those who were assigned the female gender at birth, but who identify with the male one. In Anglo-Saxon literature the term female-to-male transsexual (female-to-male transsexual or FTM in its abbreviation) is used.

Gender inconsistency or gender disagreement does not constitute a pathology per se. DMS V uses the term gender dysphoria for this "diagnosis." Gender dysphoria would be defined as the difficulty or disability, both social, occupational and other important areas of functioning, that arise from a marked inconsistency between gender of experience and the assigned gender. This experience must last at least 6 months in time, and at least 2 more criteria must be fulfilled in a list of 6 . In our Gender Identity Disorder Unit there are no uniform criteria that must be met to start a gender reassignment treatment. According to the latest guidelines from the World Professional Association for Transgender Health, Standars or Care (WPATH), proper care is needed from a mental health team.

Transgender people may undergo hormone treatment and / or gender-affirming surgeries as part of a medical transition. Trans men usually undergo bilateral mastectomy, hysterectomy with double salpingoophorectomy, and to a lesser extent a phalloplasty. WPATH recommends at least 12 months of testosterone treatment.

Trans men have a clinical indication for testosterone treatment, and there are few studies looking at its long-term effect and its effects on the breast and uterus, and the results in this regard are often contradictory. Testosterone is the key hormone in the transition of these patients for an adequate development of the secondary sexual characteristics of the desired gender. Testosterone can be administered via several routes: through daily transdermal patches and gels, weekly via subcutaneous or intramuscular injections of testosterone enanthate or testosterone cypionate, quarterly via testosterone undecanoate and orally. Regimens may be initiated at half the estimated effective dose and then titrated quickly to achieve physiological testosterone levels. Patients typically will notice signs of male sexual maturation (increased facial and body hair, muscle mass, acne, and libido), as well as amenorrhea within the first several months after initiating testosterone therapy [13]. The appropriate dose to achieve this may vary from patient to patient, but it should usually be repeated every 2 weeks to achieve good baseline levels [2]. We must not forget to provide adequate preoperative care and exams, equivalent to other type of patients, such as preoperative anesthesia visit. There are currently no specific guidelines on the perioperative care of transgender men undergoing hysterectomy; however, planning is necessary, and there are some nuances in this patient population that should be considered before proceeding to the operating room. Preoperative testing including laboratory studies, cardiac evaluation, and pulmonary function tests should be performed according to standard American Society of Anesthesiologists (ASA) guidelines taking into account the patient's comorbid medical conditions [13].

Despite the fact that there are some studies such as the one by Dizon [3] that describes the association of ovarian cancer and testosterone treatment, only one case is reported, and admits that no conclusive statements can be established in this regard. They also suggest that chronic testosterone therapy may increase the risk of endometrial and ovarian cancer. The hypothesis of increased risk that arises in the 
endometrium is based on the continuous, unopposed action of estrogens, since testosterone would be aromatized in this final molecule. Likewise, it would also be related to the ovarian formation of a polycystic aspect, although this is not shared by other publications. Similarly, there is no current evidence that testosterone treatment modifies the risk of developing cervical cancer, although there are more unsatisfactory cytologies [4]. Grynberg states in his article that long-term testosterone therapy causes abnormalities in the architecture of the ovary compatible with PCOS, both microscopically and macroscopically, as well as an increased risk of endometrial atrophy and fibrosis of the breast tissue [5, 8 , 10].

\section{Methods}

We carried out a retrospective study with 117 patients between the years 2010 and 2019. The mean age of the patients was 34,228 years (range from 21 to 56 years), with an average duration of parenteral testosterone treatment until surgery of 5.69 years. All the patients carried out a clinical-analytical followup with analysis of the values of: follicle stimulating hormone (FSH), luteinizin hormone (LH), testosterone (TST) and estradiol in blood, both preoperatively and postoperatively.

The preoperative ranges of each hormone were: FSH $0.27-121$, $\mathrm{LH}<0.07-48.8$, estradiol 29.74-183; while postoperative were: FSH 0.44-171, LH<0.07-71.33, E2 5-89.

All patients underwent a laparoscopic hysterectomy with double adnexectomy between 2010 and 2019 .

All patients were administered parenteral testosterone, in doses of 100 or $200 \mathrm{mg}$ every 21 days. The decision between one dose or the other was based mainly on the blood levels of testosterone, the degree of satisfaction with body masculinization, amenorrhea and hematocrit control, hypertension and lipid profile.

\section{Results}

The most frequent pathological result was atrophic endometrium, and multifollicular cysts were found in up to 44 patients.

The following graphs show the hormonal values of follicle-stimulating hormone (Fig. 1), luteinizing hormone (Fig. 2) and estradiol (Fig. 3) before and after surgery.

In the case of follicle-stimulating hormone ( $\mathrm{FSH})$, an increase in its levels is generally observed in patients after hysterectomy and bilateral salpingo-oophorectomy. In $76 \%$ of patients, an increase in FSH concentration was observed after the intervention, in $7 \%$ they decreased, and $17 \%$ maintained stable values (variation of $<5 \mathrm{UI} / \mathrm{L}$ ).

In the same way, although to a lesser extent, an increase in their levels is observed after the intervention. An increase in the concentration of $\mathrm{LH}$ is observed in $71 \%$ of the patients after the intervention, in $9 \%$ they decrease and in $20 \%$ they maintain stable values (variation of $<2 \mathrm{IU} / \mathrm{L}$ ). 
In this case, as might be expected, a decrease in estrogen levels is generally observed after surgical treatment. An increase in E2 concentration was observed in $22 \%$ of patients after the intervention, in $71 \%$ a decrease, and in $7 \%$ stable values were maintained (variation of $<5 \mathrm{IU} / \mathrm{L}$ ).

No significant differences or statistical association were found between the amount of testosterone administered, the preoperative analytical value of $\mathrm{FSH}$ or $\mathrm{LH}$, and the pathological finding.

The findings detected in the pathological analysis of the uterus (Fig. 4) and ovary (Fig. 5) of our study population are described below:

The most frequent pathological result was atrophic endometrium (80.3\%), finding a mean age among patients of 36.61 years and a mean duration of treatment of 6.06 .7 cases with finding of endometrium with changes of secretory type (6\%) are described, with a mean age of 32.28 years and a mean duration of treatment of 5.85 years. 15 cases of weakly proliferating endometrium (13.7\%) are described, with an average age of 28.85 years and an average duration of treatment of 5.5 years.

In $51 \%$ of the cases the pathological anatomy found in the ovaries was normal, in $37 \%$ poly-funicular ovaries and in $7 \%$ a corpus luteum was found. 5 cases with specific pathological findings are described: 1 case of mature cystic teratoma (1\%) in a 34-year-old patient and 7 years of treatment, 1 case of mucinous cystadenoma (1\%) in a 35-year-old patient, 1 case of mixed gonadal dysgenesis (1\%) in a $34-$ year-old patient with unknown duration of treatment, 1 case of adenomatoid mesothelioma (1\%) in a 51year-old patient with 9 years of treatment, and 2 cases of conventional Brenner (1\%) tumor (with areas of grade 1 cytological atypia) that curiously underwent surgery on the same day, 46 and 47 years old respectively and 6 years of previous treatment with the same amount of testosterone.

Of the 8 corpus luteum found in the ovaries, 6 of them corresponded to surgical specimens in which secretory endometriums were also found, being an expected finding already described by Loverro8. The multifollicular cysts described corresponded to patients whose endometrial analysis revealed secretory type. No case of uterine or ovarian neoplasia was found.

\section{Discussion}

In the feminine to masculine transition (FTM), also called male transgender, the primary objective is to induce virilization. To achieve masculinization in biological women, this is mainly accomplished using exogenous testosterone. Testosterone is an androgenic sex hormone responsible for male development and characteristics.

The different authors who publish on FTM can be divided into two groups, on the one hand those that defend an increased risk of endometrial and even breast cancer, a hypothesis based on the aromatization of estrogens in testosterone in menopause at the endometrial level [3]. And, on the other hand, those who defend that, under this premise, such risk increase is not possible since there is no aromatase at this level [10]. 
In our data, we did not find an increase in post surgical estradiol levels in our hysterectomized patients. We have to consider that the populations described in the literature are not uniform and that, in light of our results, surgery for excision of the internal genital tract has an effect protective as it can not be otherwise.

Moreover, those who, like us, do not find sufficient data to affirm such increased risk, rely on this same line already expressed by Perrone[7] in his article, in which he argues that parenteral administration of exogenous testosterone induces the same changes found in postmenopausal women: atrophy, a circumstance that is demonstrated in our population, being the most frequent finding in the surgical specimen (81.19\%). In fact, these results confirm those published by Loverro and Tuckerman in in vivo studies[8,9], where they suggested that high doses of testosterone would induce endometrial atrophy by inhibiting cell proliferation. Moreover, most of studies agree that long testosterone exposure is related to atrophic or inactive endometrium, most recently said by Khalifa et al[12]. We have only found one study against this argument, where they describe active endometrium in most of its patients [11]. Nevertheless, the dose of testosterone is not specified.

Although a specific analysis of ovarian tissue was not performed in our series, the finding of multifollicular cysts (31.62\%) would also support the theory that testosterone induces changes in the ovarian parenchyma such as those that occur normally in the polycystic ovary[6].

Despite the fact that our data and what exists in the literature coincide in their results, there is still a need for longer-term studies on the effects of parenteral testosterone. It is indisputable that such group is a good data provider, since therapy and follow-up are life-long.

\section{Conclusions}

Despite the hypothesis raised in the introduction and taking the cited articles to date as references, we cannot conclude with our data that there are relevant pathological changes in patients with chronic use of parenteral testosterone.

There is no case of endometrial cancer among our patients. There are no a priori differences in age or amount of testosterone administered and findings of a specific endometrial type. We have only found 37 cases of multifollicular cysts in the analysis of the surgical pieces, which represents less than half of the cases presented. Such fact does not allow us to establish the statement that testosterone induces polycystic ovary-type ovarian changes $[8,10]$, and, although it is highly probable, a greater number of studies are needed to clarify this finding.

\section{Declarations}

\section{We declare no conflict of interests.}


This research did not receive any specific grant from funding agencies in the public, commercial, or notfor-profit sectors.

\section{Funding}

This research received no external funding.

Author Contributions: Conceptualization, MMP, JSJL; Methodology, MMP, JSJL, MAV, E.G.-M., MPER, LSM; Software, MMP, MAV, MPER; Formal Analysis, MMP, JSJL; Investigation. MMP, JSJL, MAV; Writing Original Draft Preparation, MMP, MPER, JSJL, LSM; Writing - Review \& Editing, MMP, MAV, E.G.-M., MPER, JSJL. All authors have read and agreed to the published version of the manuscript.

Institutional Review Board Statement: The study was conducted according to the guidelines of the Declaration of Helsinki, and approved by the Institutional Ethics Committee of Malaga (protocol code $1201 / 2021$ and date of approval 2021, March 12).

Informed Consent Statement: Patient consent was waived due to the exemption authorized by the Ethics Committee because it was a retrospective study with a high number of patients and the data collected was anonymized.

Data Availability Statement: No new data were created or analyzed in this study. Data sharing is not applicable to this article.

Conflicts of Interest: The authors declare no conflict of interest.

\section{Corresponding author}

Correspondence to Jesus Salvador Jimenez Lopez

\section{Consent for publication}

No individual information is shared, and consent for publication is not applicable.

\section{References}

[1] S. Gupta, K.L. Imborek, M.D. Krasowski, Challenges in Transgender Healthcare: The Pathology Perspective, Lab. Med. 47 (2016) 180-188. doi: 10.1093/labmed/Imw020.

[2] E. Moore, A. Wisniewski, A. Dobs, Endocrine treatment of transsexual people: a review of treatment regimens, outcomes, and adverse effects. J. Clin. Endocrinol. Metab. 88 (2003) 3467-3473.

[3]D.S. Dizon, T. Tejada-Berges, S. Koelliker, M. Steinhoff, C.O. Granai, Ovarian cancer associated with testosterone supplementation in a female-to-male transsexual patient, Gynecol. Obstet. Invest. 62 (2006) 226-228. 
[4] J. Feldman, Preventive care of the transgender patient, in: R. Ettner, S. Monstrey, E Coleman (Eds), Principles of Transgender Medicine and Surgery, Routledge, New York, 2016, pp. 64-99.

[5] S.M. Peitzmeier, S.L. Reisner, P. Harigopal, J. Potter, Female-to-male patients have high prevalence of unsatisfactory Paps compared to non-transgender females: implications for cervical cancer screening, J. Gen. Intern. Med. 29 (2014) 778-784. doi: 10.1007/s11606-013-2753-1.

[6] M. Grynberg, R. Fanchin, G. Dubost, et al, Histology of genital tract and breast tissue after long-term testosterone administration in a female-to-male transexual population, Reprod. Biomed. Online. 20 (2010) 553-558. doi: 10.1016/j.rbmo.2009.12.021.

[7] A.M. Perrone, S. Cerpolini, N.C. Maria Salfi, et al, Effect of long-term testosterone administration on the endometrium of female-to-male (FtM) transsexuals, J. Sex. Med. 6 (2009) 3193-3200. doi: 10.1111/j.1743-6109.2009.01380.x.

[8] G. Loverro, L. Resta, M. Dellino, et al, Uterine and ovarian changes during testosterone administration in young female-to-male transsexuals, Taiwan. J. Obstet. Gynecol. 55 (2016) 686-691. doi: 10.1016/j.tjog.2016.03.004.

[9] E.M. Tuckerman, M.A. Okon, T.C. Li, S.M. Laird, Do androgens have a direct effect on endometrial function? An in vitro study, Fertil. Steril. 74 (2000) 771-779.

[10] P.M. Baxendale, M.J. Reed, V.H. James, Inability of human endometrium or myometrium to aromatize androstenedione, J. Steroid. Biochem. 14 (1981) 305-306.

[11] Grimstad FW, et al. Am J Obstet Gynecol. 2019 Mar;220(3):257.e1-257.e7.

[12] Khalifa MA, Toyama A, Klein ME, Santiago V. Int J Gynecol Pathol. 2019 Nov;38(6):520-527.

[13] C. Ferrando, Comprehensive care of the transgender patient. ISBN: 978-0-323-49642-1.

\section{Figures}




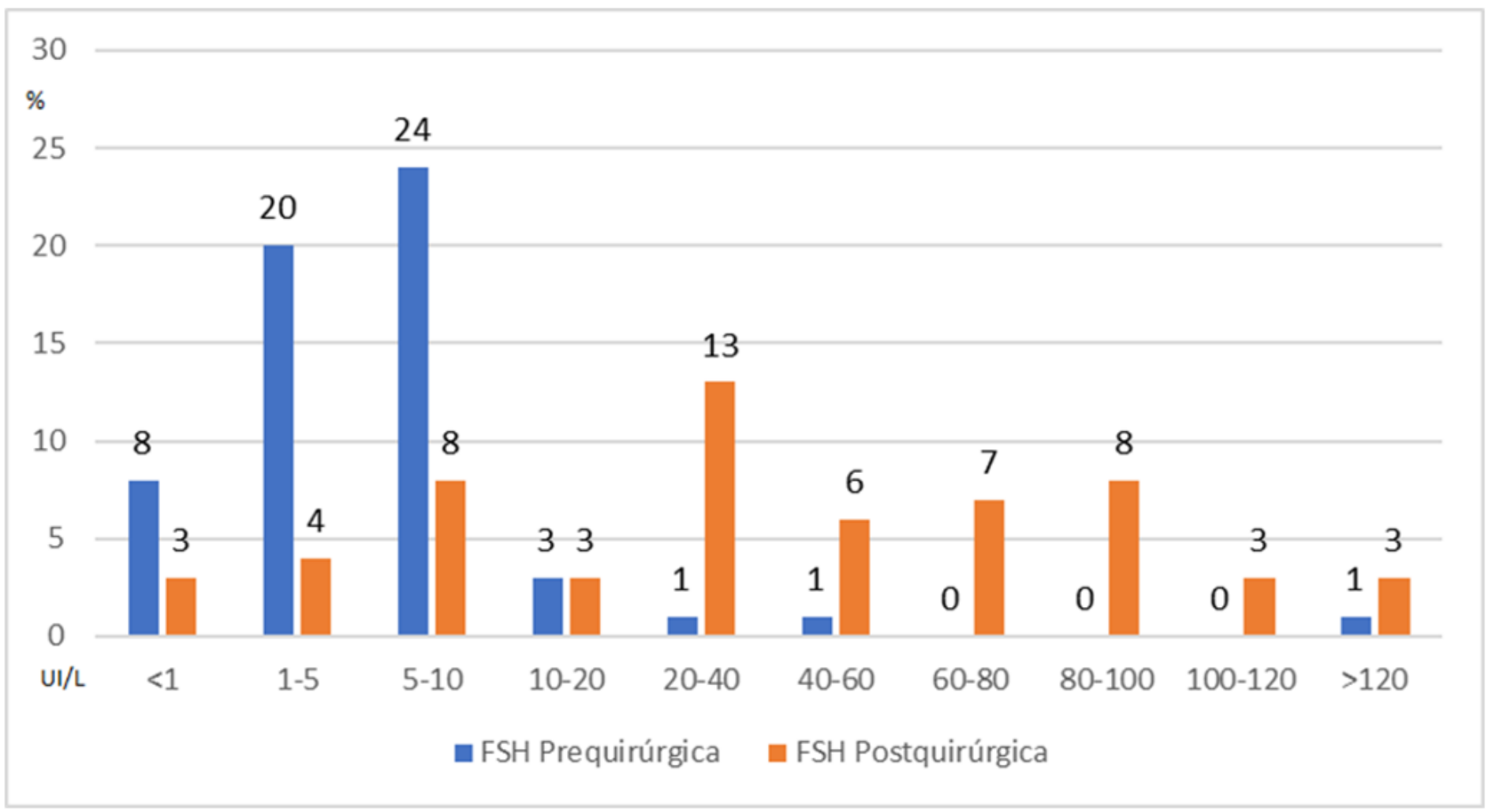

Figure 1

Follicle-stimulating hormone (FSH) value in IU / L before and after surgical treatment

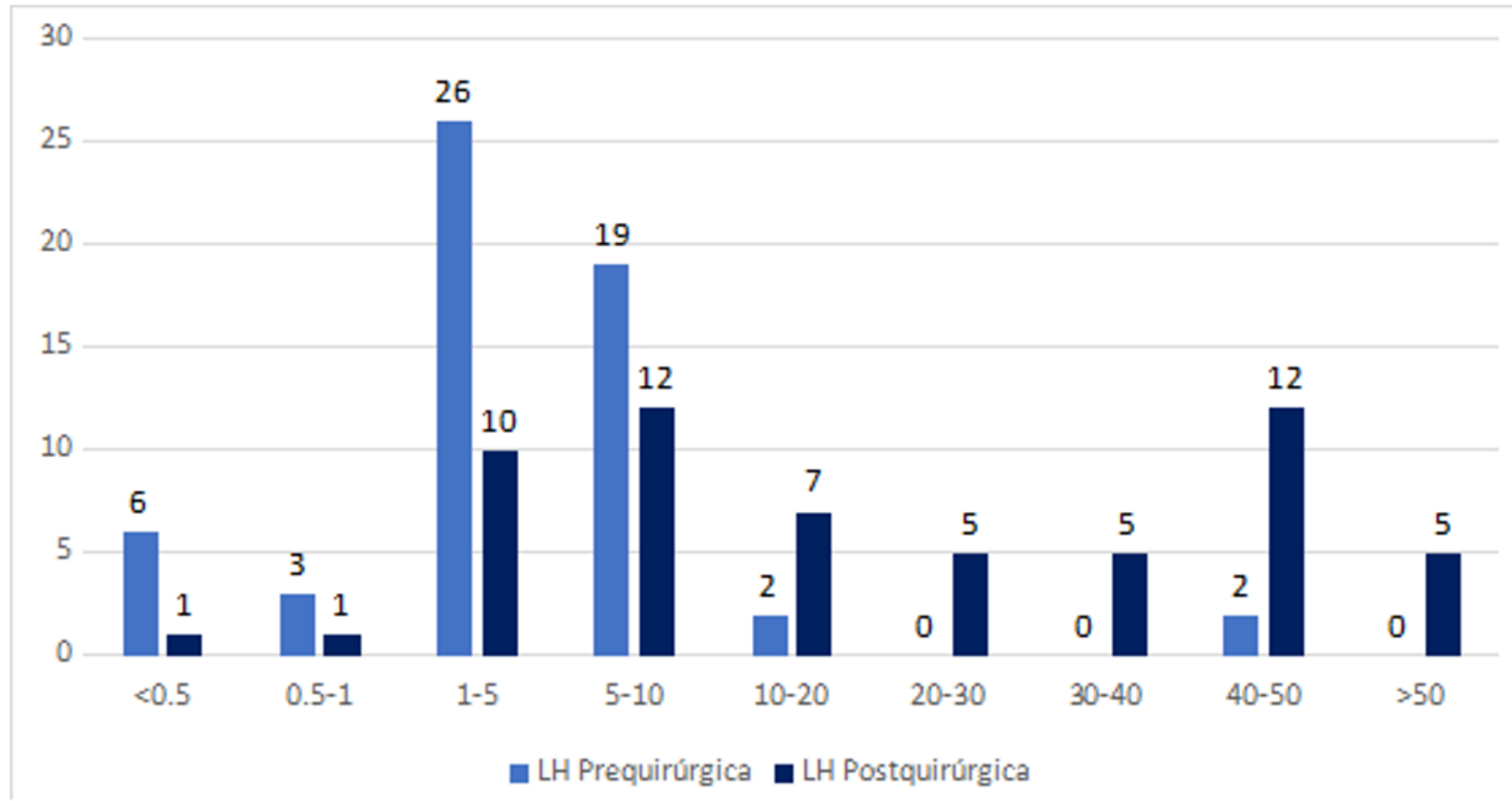

Figure 2 
Luteinizing Hormone (LH) Values in IU / L before and after surgical treatment

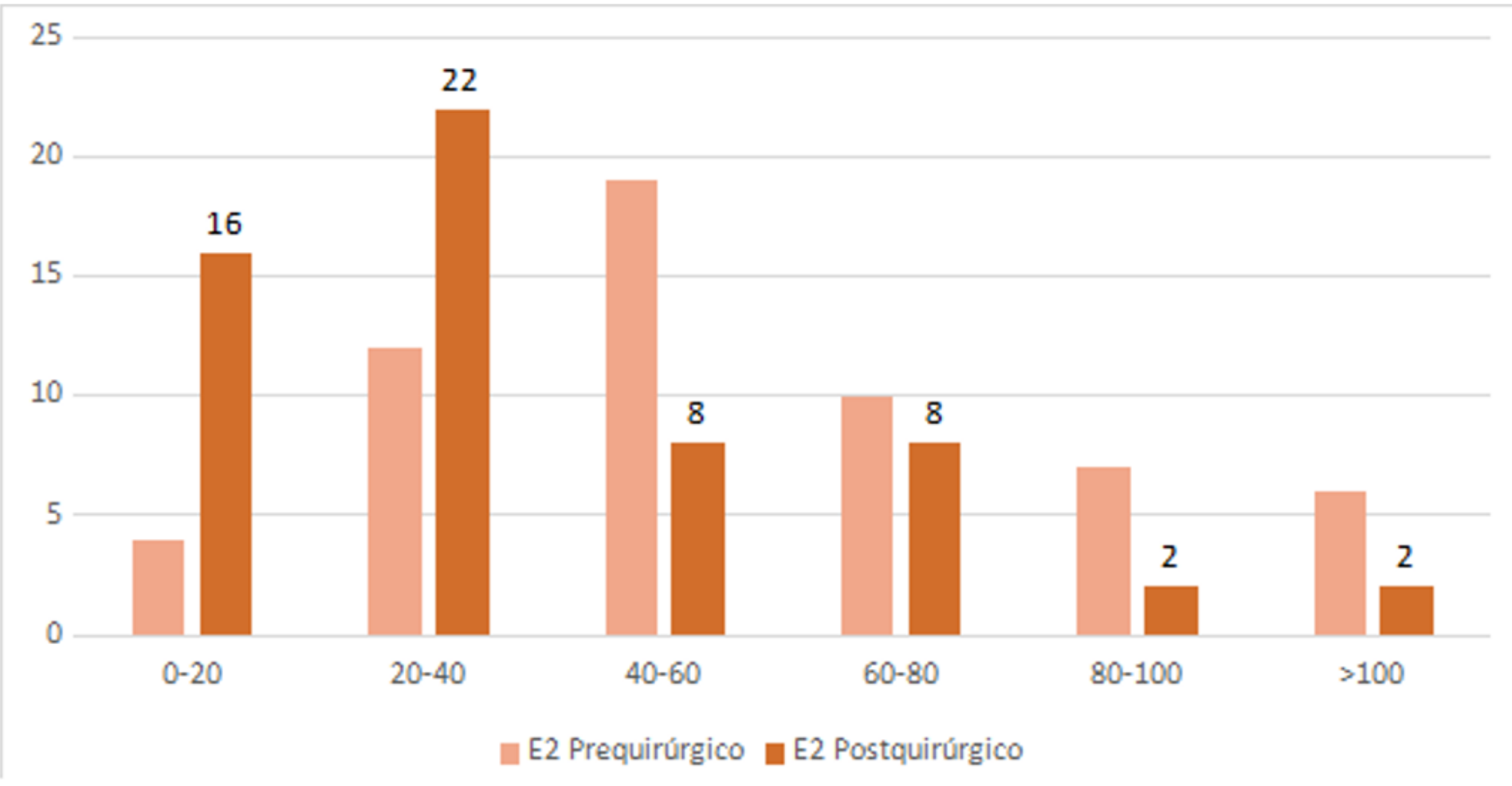

\section{Figure 3}

Estradiol (E2) values in IU / $\mathrm{L}$ before and after surgical treatment Anatomopathological study of the uterus

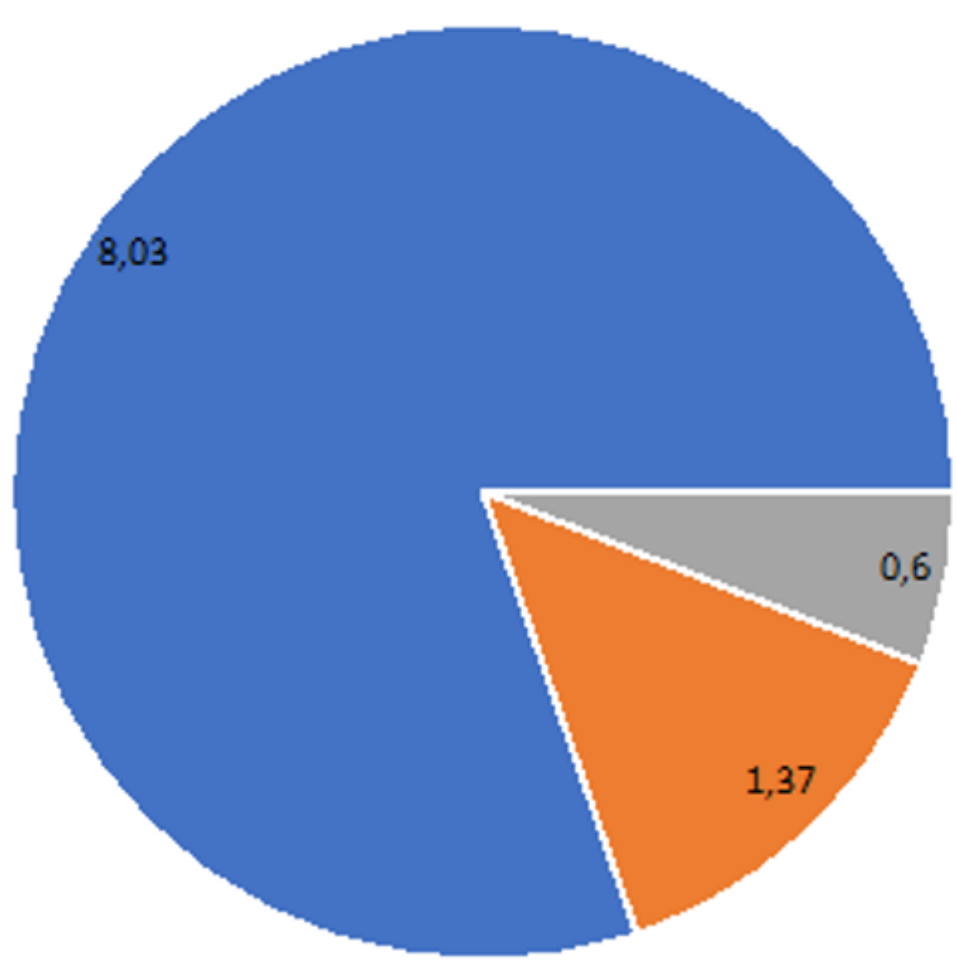

- Atrophic Endometrium 80,3\%

a Proliferat ing Endometrium 13,7\% - Secretory Endometrium 6\% 


\section{Figure 4}

Anatomopathological analysis of the hysterectomy surgical pieces

\section{Anatomopathological study of the ovaries}

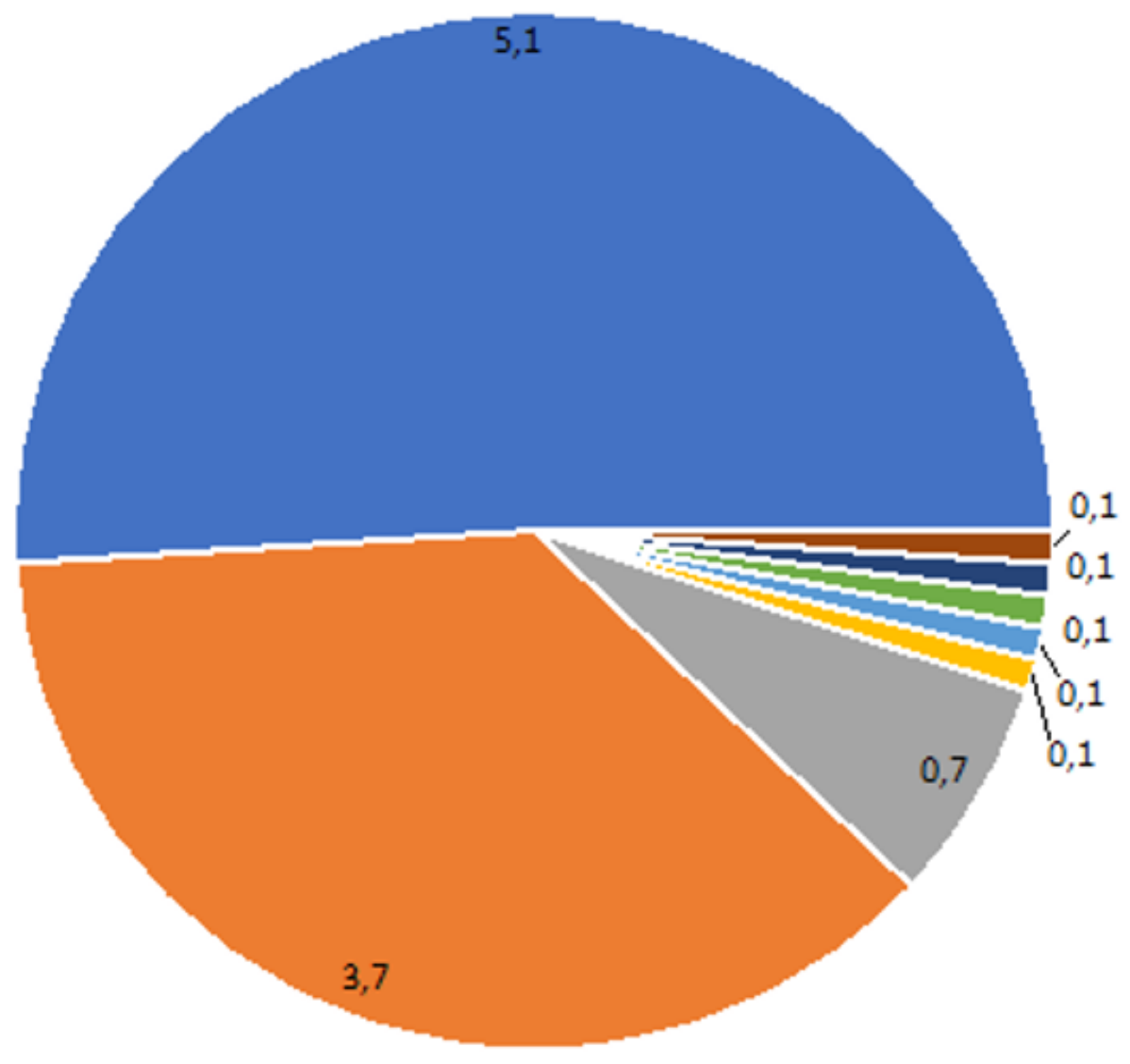

- Normal ovaries $51 \%$

- Poly-follicular ovaries $37 \%$

Corpus luteum $7 \%$

- Mucinous cystadenoma $1 \%$

- Mature cyst ć teratoma $1 \%$

nixed gonadal dysgenesis $1 \%$

- Brenner tumor $1 \%$

- Adenomatoid mesothelioma $1 \%$

\section{0,1}

口

Figure 5

Pathological analysis of the double salpingoophorectomy surgical pieces. 